\title{
The importance of monitoring vergence eye movements for solutions using virtual technologies
}

\author{
Marek Piszczek ${ }^{*}$, Mateusz Pomianek ${ }^{1}$, Marcin Maciejewski ${ }^{1}$, Leon Jodlowski ${ }^{1}$ and Piotr Krukowski ${ }^{2}$ \\ ${ }^{1}$ Institute of Optoelectronics, Military University of Technology, Kaliskiego 2, 00-908 Warszawa, \\ ${ }^{2}$ RemmedVR sp. z o.o., Domaniewska 47/10, 02-672 Warszawa
}

Received May 14, 2020; accepted June 24, 2020; published June 30, 2020

\begin{abstract}
Eyesight monitoring systems are of particular interest to virtual technology research teams and concern mainly three key aspects: physiological, technical and functional. The first one is to solve the socalled conflict of vergence and accommodation. In contrast to typical gaming applications, e.g. vision therapy requires the ability to smoothly transfer attention between the points of near and far. The technical aspect is primarily the search for new measurement methods, which is particularly important in the case of mobile goggles with limited computing capabilities of the processor. The last aspect concerns the possibility of creating effective HMI (Human Machine Interface) solutions.
\end{abstract}

Eyesight is the dominant channel of information acquisition by humans and enables them to function efficiently in surrounding reality. Particularly noteworthy is stereoscopic vision, which is one of the basic factors allowing to assess the position of objects in the threedimensional space surrounding the observer. Therefore, recently, some researchers have been particularly interested in sight tracking, among others, because of the possibility of creating solutions for sight diagnostics and therapy as well as broadening the ways of human-machine communication. Thus, the key issue is the monitoring of vision vergence, and the research is focused on physiological, technical, and functional aspects.

The physiological aspect is, above all, the ability to correctly reproduce the mechanism of spatial vision in virtuality. From the point of view of the human visual system, the eye's vergence movements are closely linked to accommodation. Unfortunately, the majority of commercially available 3D VR/AR goggles are solutions that use only the vergence mechanism. In the literature this phenomenon is known as Vergence-Accommodation Conflict (VCA) (Fig. 1). Incorrect functioning of the mechanism of accommodation in relation to the vergence stimulus causes discomfort in the reception of the content presented by using virtual space. This problem becomes particularly important for the development of VR goggles dedicated to the therapy of amblyopia when the patient's involvement in computer games is used [1].

*E-mail: marek.piszczek@wat.edu.pl

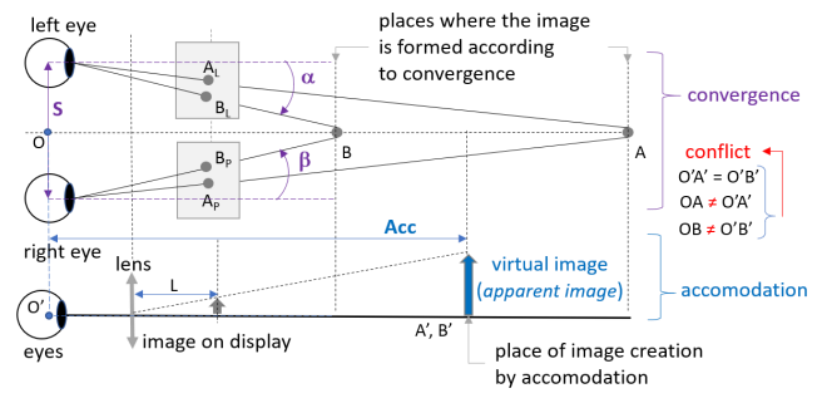

Fig. 1. Vergence Accommodation Conflict in 3D VR/AR goggles.

An experimental laboratory stand for testing accommodation issues is shown in Fig. 2. Section (B) synthesizes (in the range of $30 \mathrm{~cm} \div \infty$ ) the correct accommodation stimulus (Acc - the distance of the apparent image from the observer) based on a geometric analysis of $\alpha, \beta$ and stereoscopic eye base $S$ (see Fig. 1):

$$
A_{C C}=f(S, \alpha, \beta) \text {. }
$$

Sections (C and D) are responsible for the analysis of accommodation response according to the retinoscopy method [2]. The vision/projection system acting as an artificial eye (section A - using electrically controlled optics EL-10-30-TC) has been used to provide objective measurements.

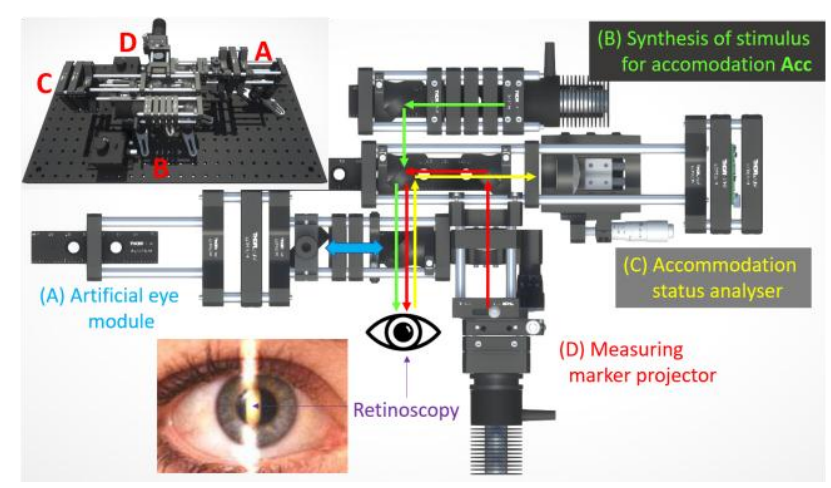

Fig. 2. Measurement stand for accommodation tests. 
VR glasses therapy is a gaming solution that requires the user to perform simple manual activities and follow objects visually. Therapeutic content differs from the required stimulation for the right or left eye. In order for the therapy process to proceed properly, therapeutic goggles must synthesize appropriately corrected accommodation images for both near and far points of convergence. It is also necessary to control the state of accommodation and vergence during eye therapy. A general diagram of this process is shown in Fig. 3.

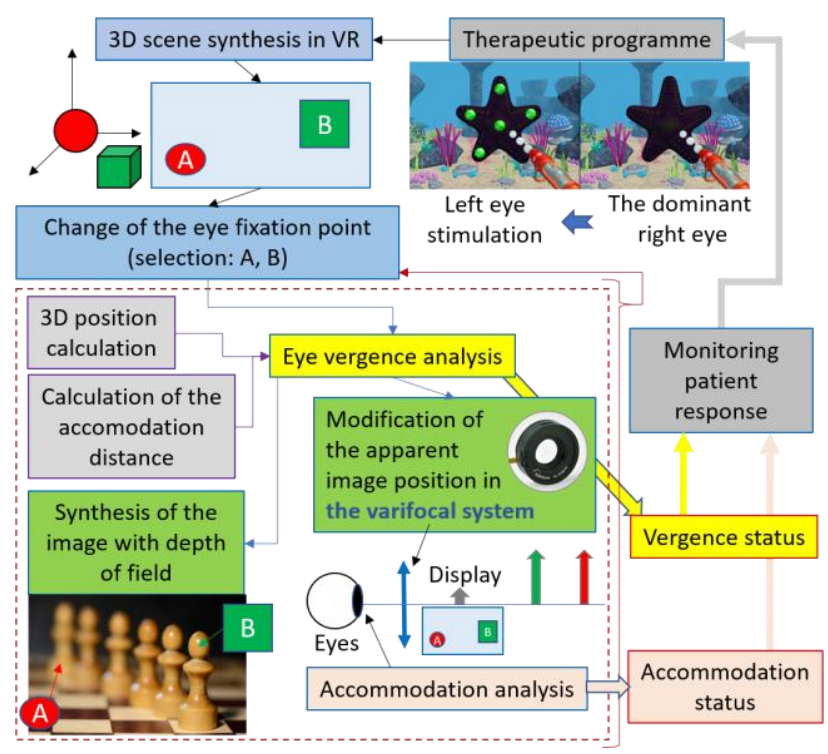

Fig. 3. RemmedVR device for home vision therapy.

The technical aspect is mainly hardware and software methods of measuring eyes vergence. Currently, the most popular solution is eye-tracking based on the analysis of images from the vision system [3]. The basic video analysis comes down to classical methods of image processing related to the search for the pupil area and then analysis of its shape. However, such measurement methods often require more computing power associated with the need to use an operating system and dedicated libraries. The implementation of such solutions in mobile goggles becomes problematic because most of the computing power must be directed to support other elements of virtual technology: rendering VR scenes or monitoring the user's activity in local space. Therefore, there is a need to look for other solutions. One possibility is to use a different type of sensors, delegate measurement and analysis tasks to relatively simple controllers and deliver highly processed data to goggles. For monitoring eyes vergence this alternative can be $2 \mathrm{D}$ profile sensors or optical scanning systems based on 2D MEMS mirrors [4]. Moving from the acquisition and processing of a twodimensional data set $\left(>10^{5}\right.$ pixles $)$ to fewer onedimensional data $\left(\sim 10^{3}\right.$ points $)$ significantly reduces calculation requirements. The common feature of all these methods (image or signal analysis) is estimating the pupil centre of the eyes. A three-dimensional ophthalmic model can be developed during calibration. On this basis, with the knowledge of vergence momentary angles, it is possible to determine the points of fixation of the eyes in 3D space. Working with one-dimensional data allows to obtain comparable measurement resolutions at shorter refresh rates. For solutions with MEMS modules the frequencies can be $\sim 1 \mathrm{kHz}$ and for profile sensors $\sim 3 \mathrm{kHz}$.

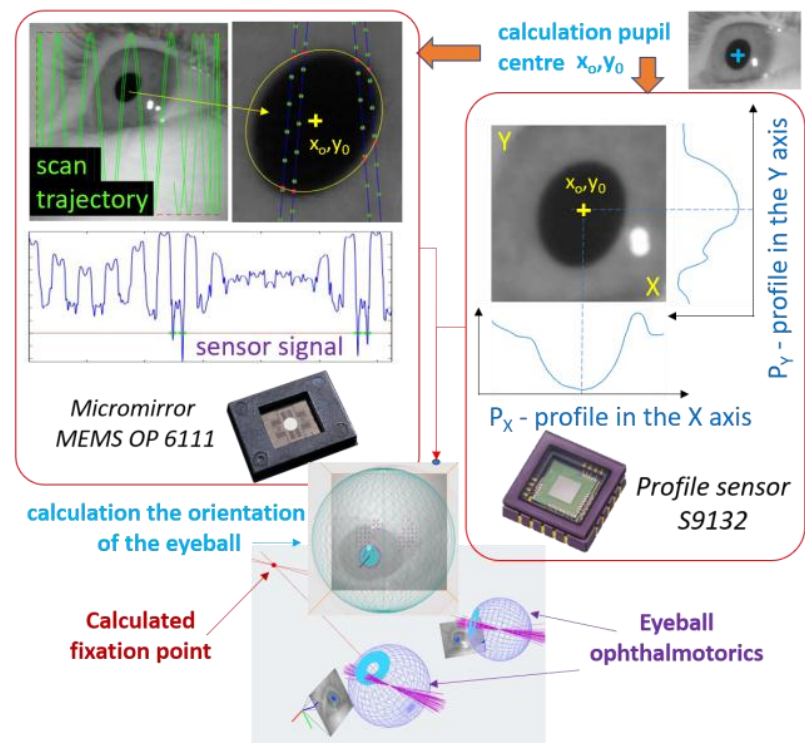

Fig. 4. Calculation of eyeball orientation and eye fixation point.

In our stand (Fig. 4) a 2D profile analysis is tested using the Hamamatsu S9132. The pupil position is estimated by approximating inverted profiles from the $\mathrm{X}$ and $\mathrm{Y}$ axes with a normal distribution, where pupil coordinates are their mean values.

In the case of MEMS 2D micromirror eye scanning, the OP 6111 system is used. Subsequent measuring points of the detected signal are arranged according to Lissajous figures with known coordinates $(x, y)$. To estimate the pupil centre it is enough to determine the points near the edge of the pupil. Approximation of the distribution of these points with, e.g. an ellipse, allows to determine the desired parameters:

$$
A x^{2}-B x y+C x^{2}-D x-E y+F=0 \rightarrow\left(x_{0}, y_{0}\right) .
$$

The great possibility of modifying the operating parameters of the system with 2D MEMS (angular range control, sampling resolution), as well as the ability to work with a 2D profile sensor (as a pupil pre-positioning system), indicates the possibility of estimating the ocular vergence angles at $1 \mathrm{arcmin}$. 
The third important issue concerning the monitoring of eye vergence movements (knowledge of the momentary values of the fixation point) is related to the practical possibilities of using this type of data. This concerns primarily the extension of the HMI in terms of interaction possibilities. Thanks to virtual technologies, traditional physical activities can be extended through visual interaction. This may involve handling the GUI of the application or interacting with other types of objects, which will result in a specific reaction, e.g. activating these objects or displaying information about them (Fig. 5).

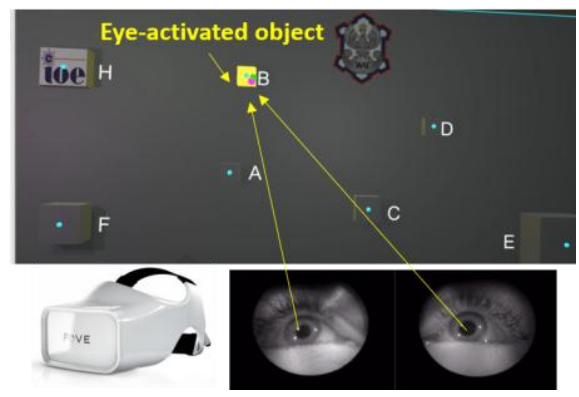

Fig. 5. Visual interaction in a virtual environment using FOVE HMD.

The experiments also show that it is possible to explore the physical world "indirectly" through the "eye-gaze" HMI. Such a solution, for example, can be based on the integration of a vision measurement system with a virtual environment. A calibrated stereoscopic vision system provides not only images for the left and right eye but also information about their spatial relationships. This allows images to be integrated into a virtual scene taking into account the scale of conversion of the stereoscopic base of the $S x$ vision system to the stereoscopic base of the $S$ observer's eyes:

$$
\begin{aligned}
& S=S_{X} \cdot \text { scale } ; \\
& \text { micro } \rightarrow \text { scale }>1 ; \text { macro } \rightarrow 0<\text { scale }<1
\end{aligned}
$$

The observer is able to visually perceive the depth of the image material (provided by the stereo-vison system), regardless of the imaging scale used. Thus, one can transfer one's visual attention to the objects of the actual scene at different distances. Which of the objects in the above scene is currently in the area of interest is determined by the fixation point. This, in turn, is determined by measuring the eye vergence. From the point of view of the vision system and stereoscopic analysis, the direction of each eyeball indicates the homology points in the left and right image. This is sufficient for the calibrated vision system to determine the three-dimensional position of such a pair of points.
Analyses of a set of such sequentially indicated points make it possible to perform spatial analyses: measurements of distances, angles, surface fields or volumes (Fig. 6).

Visual interaction is not only a new/alternative way of contact. In many cases it can offer a much higher speed of human-machine communication $(10 \div 50 \%)$, shorter user response times $(0.1 \div 0.5 \mathrm{~s})$ and in other cases it can be the only available way to operate the application interface (when manual and verbal solutions are not available).

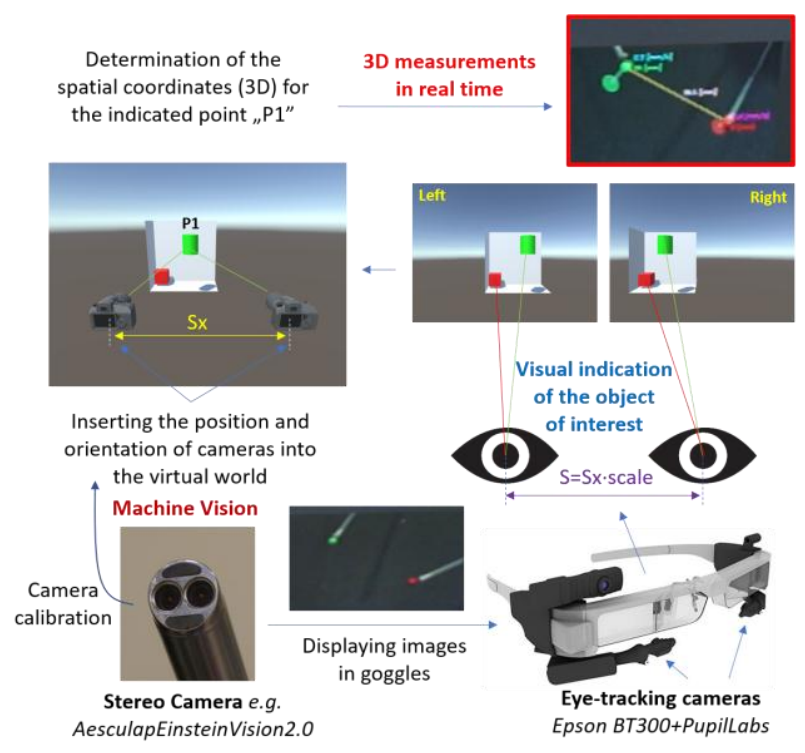

Fig. 6. Visual interaction with real images using Epson (AR) HMD.

The above considerations show how important optoelectronic systems related to the analysis of eye regeneration are for virtual technologies. The search for satisfactory solutions for VCA and methods of tracking eyes with reduced computational requirements will allow to develop an effective therapeutic tool for optometrists. What is more, the possibility of visual interaction is a new tool for cyberphysical systems.

This work was supported by the National Centre for Research and Development, project POIR.01.01.01-001201/18, "RemmedVR device for home vision therapy".

\section{References}

[1] J.D. Bayliss et al., Lazy Eye Shooter: a Novel Game Therapy for Visual Recovery in Adult Amblyopia, IEEE International Games Innovation Conference 2012

[2] Y.L. Chen., L. Shi, J.W.L. Lewis, M. Wang, Photonics 1, 303 (2014).

[3] W. Fuhl, M. Tonsen, A. Bulling, E. Kasneci, Mach. Vis. Appl. 27(8), 1275 (2016), doi: 10.1007/s00138-016-0776-4.

[4] N. Sarkar, D. Strathearn, G. Lee, M. Olfat, A. Rohani, R.R. Mansour, 18th International Conference on Solid-State Sensors, Actuators and Microsystems (Transducers 2015), pp. 855-858 (2015), doi: 10.1109/TRANSDUCERS.2015.7181058. 